\title{
Cytogenetic analysis for research and services
}

\author{
Sultana MH Faradz* \\ Center for Biomedical Research, Faculty of Medicine, Diponegoro University
}

DOI: http://dx.doi.org/10.19106/JMedScieSup004804201631

\section{ABSTRACT}

The correct number of human chromosome, 46, was first recognized by Tjio and Levan in 1956. Cytogenetic analyses are commonly performed to determine both structural and numerical chromosome aberration, whilst changes in chromosomes might lead to birth defects, syndromes, and cancer. Several chromosomal aneuploidy syndromes were identified after the establishment of various chromosome banding techniques in late 1960s, and specific cell culture medium was found to express fragile site in the beginning of 1970s. Since then, inherited Fragile X Mental Retardation syndrome could be diagnosed. However, some female permutation cases were still often misdiagnosed. Further molecular analysis had solved this problem by revealing more CGG repeats in the promoter region FMR1 gene, which is related to the expression of fragile site and the severity of the diseases.

In cases of Disorder of Sex Development (DSD), early gender assignment and reconstruction surgery were challenged due to the dilemma of gender identity development later in life. Cytogenetic analysis for the first-line gender assignment is important in newborn with DSD. Proper diagnosis with hormonal and mutation analysis should be elucidated to avoid medical, psychological, and social issues in adult life. The most frequent genetic cases in our clinical experiences were Androgen Insensitivity Syndrome and Congenital Adrenal Hyperplasia. For example, without cytogenetic analysis, female Complete Androgen Insensitivity Syndrome (CAIS) with primary amenorrhea as the main symptom was frequently misdiagnosed as inguinal hernia, due to the location and size of testicles.

Diagnosis and treatment of several leukemias and lymphomas, as well as some solid tumors, depend on cytogenetic analyses to demonstrate consistent, specific chromosomal aberrations. Chromosome analysis in hematologic malignancy is indicated to support diagnosis, select therapy regimen, and determine prognosis. Specific chromosome translocations have been identified in hematologic malignancy. The breakpoints of several of these translocations have been cloned. Several loci of oncogene have also been identified and 
sequenced. Molecular genetic analysis will replace cytogenetic analysis and shift the requirement for studying cells in metaphase. Therefore, chromosome analysis in genetic disease and cancer should be attained with advanced molecular techniques, such as Fluorescence In-Situ Hybridization (FISH) and microarray $\mathrm{CGH}$ analysis. Cytogenetic analysis is still useful and applicable in genetic disease diagnosis, sexual assignment, and hematologic malignancy in the laboratory with minimal equipments. Molecular analysis as a part of health care services in Indonesia has been limited in research centers in university setting; therefore, a comprehensive diagnosis with genetic analysis is often improbable.

Keywords: cytogenetics, chromosome, syndrome, DSD, hematologic malignancy 